DESY 03-189

November 2003

\title{
The role of $\sigma\left(e^{+} e^{-} \rightarrow\right.$ hadrons $)$ in precision tests of the Standard Model
}

\author{
F. Jegerlehner ${ }^{\S}$ \\ DESY Zeuthen \\ Platanenallee 6, D-15738 Zeuthen, Germany
}

$\S$ Invited talk at SIGHAD03: Worskhop on Hadronic Cross Section at Low Energy, Pisa, Italy, October 8-10, 2003. Work supported in part by TMR, EC-Contract No. HPRN-CT-2002-00311 (EURIDICE) 


\title{
The role of $\sigma\left(e^{+} e^{-} \rightarrow\right.$ hadrons $)$ in precision tests of the Standard Model
}

\author{
F. Jegerlehner ${ }^{a *}$ \\ ${ }^{a}$ Deutsches Elektronen-Synchrotron (DESY), Platanenallee 6, D-15738, Zeuthen, Germany
}

I present a summary of recent developments as they have been discussed in the many interesting contributions to this workshop (SIGHAD03 at Pisa).

\section{1. $\alpha\left(M_{Z}\right)$ IN PRECISION PHYSICS}

Hadronic cross section measurements in $e^{+} e^{-}$ annihilation are an indispensable input for the estimation of the non-perturbative hadronic contribution the shift in the fine structure constant, one of the fundamental input parameters for making precise theoretical predictions for electroweak processes in standard electroweak theory (SM). The errors of the experimental data directly affect and limit the precision at which theoretical predictions can be made. Thus the uncertainties of the hadronic contributions to the effective fine structure constant $\alpha(E)$ ( $E$ the energy scale) are a serious problem in electroweak precision physics 11. At present the most precisely known parameters are $\alpha, G_{\mu}$ and $M_{Z}$ and typical (pseudo-) observables for which precise predictions are possible are $\sin ^{2} \Theta_{f}, v_{f}, a_{f}$ defined via the $Z \bar{f} f$ neutral current vertex ( $f$ any lepton or quark) or the gauge boson parameters $M_{W}, \Gamma_{Z}, \Gamma_{W}, \cdots$. However, it is not the low energy fine structure constant $\alpha$ itself, but the effective value $\alpha\left(M_{Z}\right)$ which is the appropriate input parameter for predicting properties of the weak gauge bosons $Z$ and $W$. The two are related by non-perturbative physics, to be discussed below. Present accuracies of the basic parameters are the following: $\frac{\delta \alpha}{\alpha} \sim 3.6 \times 10^{-9}, \frac{\delta G_{\mu}}{G_{\mu}} \sim 8.6 \times 10^{-6}$, $\frac{\delta M_{Z}}{M_{Z}} \sim 2.4 \times 10^{-5}, \frac{\delta \alpha\left(M_{Z}\right)}{\alpha\left(M_{Z}\right)} \sim 1.6 \div 6.8 \times 10^{-4}$ (present), while $\frac{\delta \alpha\left(M_{Z}\right)}{\alpha\left(M_{Z}\right)} \sim 5.3 \times 10^{-5}$ is what will be required for precision physics at a future linear collider (like TESLA). Note the dramatic loss in acuracy by five orders of magnitude in going from $\alpha=\alpha(0)$ to $\alpha\left(M_{Z}\right)$. A typical uncer-

\footnotetext{
*Work supported in part by TMR, EC-Contract No. HPRN-CT-2002-00311 (EURIDICE)
}

tainty in $\alpha$ is $\delta \Delta \alpha\left(M_{Z}\right)=0.00036$ which contibutes to the uncertainty $\delta \sin ^{2} \Theta_{\text {eff }}=0.00013$, which affects substantially the Higgs mass upper bound which derives to a large extent from the $\sin ^{2} \Theta_{\text {eff }}^{\ell}(\ell=e, \mu, \tau)$ measurements. The $\mathrm{LEP} / \mathrm{SLD}$ result at present is $\sin ^{2} \Theta_{\text {eff }}^{\ell}=\frac{1}{4}(1-$ $\left.\frac{v_{\ell}}{a_{\ell}}\right)=0.23148 \pm 0.00017$. Most precision observables also receive perturbative QCD (pQCD) contributions, the accurate evaluation of which is depending on a precise knowledge of the QCD parameters $\alpha_{s}, m_{c}, m_{b}, m_{t}$. Corresponding efforts in pQCD, lattice QCD, chiral perturbation theory and/or exploiting sum rules are mandatory in this respect. An important set of quantities which directly depend on $\Delta \alpha$ are the mixing parameters $\sin ^{2} \Theta_{i}$ obtained from $\alpha, G_{\mu}$ and $M_{Z}$ via the generalized Sirlin relation:

$\sqrt{2} G_{\mu} M_{Z}^{2} \sin ^{2} \Theta_{i} \cos ^{2} \Theta_{i}=\frac{\pi \alpha}{1-\Delta r_{i}}=\frac{\pi \alpha\left(M_{Z}\right)}{1-\Delta \bar{r}_{i}}$

with effective fine structure constant

$\alpha(M)=\frac{\alpha}{1-\Delta \alpha(M)}$.

Various definitions of $\sin ^{2} \Theta_{i}$, which coincide at tree level (i.e., for $\Delta r_{i} \sim 0$ ) are the following: from weak gauge boson masses, from electroweak gauge couplings and from the neutral current couplings of the charged fermions one defines $\sin ^{2} \Theta_{W}=1-\frac{M_{W}^{2}}{M_{Z}^{2}}, \sin ^{2} \Theta_{g}=e^{2} / g^{2}$ or $\sin ^{2} \Theta_{f}=\frac{1}{4\left|Q_{f}\right|}\left(1-\frac{v_{f}}{a_{f}}\right)(f \neq \nu)$, respectively. The radiative correction $\Delta r_{i}=$ $\Delta r_{i}\left(\alpha, G_{\mu}, M_{Z}, m_{H}, m_{f \neq t}, m_{t}\right)$ distinguishing the different definitions depend on all SM parameters and thereby provide an indirect constraint on $m_{t}$, $m_{H}$ or possible new physics, which also would 
contribute. The leading corrections $\Delta \alpha$ and $\Delta \rho$ (leading $m_{t}$-dependence) enter in the form $\Delta r_{i}=$ $\Delta \alpha+\Delta \bar{r}_{i}=\Delta \alpha-f_{i}\left(\sin ^{2} \Theta_{i}\right) \Delta \rho+\Delta r_{i}$ rem, exhibiting an universal dependence on $\Delta \alpha$. The predictions for $M_{W}, A_{L R}, A_{F B}^{f}, \Gamma_{f}, \cdots$ are thus directly affected by the error $\delta \Delta \alpha$ which translates into uncertainties $\delta M_{W}, \delta \sin ^{2} \Theta_{i}$, etc. ${ }^{2}$. The indirect Higgs boson mass "measurement" yields $m_{H}=96_{-30}^{+60} \mathrm{GeV}\left(e^{+} e^{-}\right.$-data based evaluation of $\Delta \alpha)^{3}$. For further details I refer to [123.

\section{RECENT EXPERIMENTAL AND THEORY INPUT}

Recent advances/issues in the evaluation of the hadronic vacuum polarization (VP) are based on the following results:

- Updated results from the precise measurements of the processes $e^{+} e^{-} \rightarrow \rho \rightarrow \pi^{+} \pi^{-}$, $e^{+} e^{-} \rightarrow \omega \rightarrow \pi^{+} \pi^{-} \pi^{0}$ and $e^{+} e^{-} \rightarrow \phi \rightarrow K_{L} K_{S}$ performed by the CMD-2 collaboration have appeared recently [45]. The update was necessary due to an overestimate (partally missing subtraction of VP effect in the Bhabha- and $\mu \mu$-channels) of the integrated luminosity in the previous analysis which was published in 2002 [6]. A more progressive error estimate (improving on radiative corrections, in particular) allowed a reduction of the systematic error from $1.4 \%$ to 0.6 $\%$. Subtracting the vacuum polarization systematically at time-like scales leads to lower crosssections. Some other CMD-2 and SND data at energies $E<1.4 \mathrm{GeV}$ have become available as well. CMD-2 now is in good agreement with SND results on the $4 \pi$ channels.

- Before in 2001 BES-II published their final $R$ data which, in the region $2.0 \mathrm{GeV}$ to $5.0 \mathrm{GeV}$, allowed to reduce the previously huge systematic errors of about $20 \%$ to $7 \%$ 78.

- After 1997 precise $\tau$-spectral functions became available 9101112 which, to the extent that flavor $S U(2)$ in the light hadron sector is a good symmetry, allows to obtain the iso-vector part of the $e^{+} e^{-}$-cross section [13. This possibility has

\footnotetext{
${ }^{2}$ see plot files: w03_sef2_theta.eps, w03_mw.eps at lepewwg . web.cern.ch/LEPEWWG/plots/winter2003/

${ }^{3}$ If one would base the evaluation on $\tau$-data instead, the central value would be shifted by about $\delta m_{H} \sim-10 \mathrm{GeV}$.
}

first been exploited in the present context in [14]. First results on $\tau$-spectra from BELLE have been presented at this meeting [15].

- With increasing precision of the low energy data it more and more turned out that we are confronted with a serious obstacle to further progress: in the region just above the $\omega-$ resonance, the iso-spin rotated $\tau$-data, after being corrected for the known iso-spin violating effects, do not agree with the $e^{+} e^{-}$-data at the $10 \%$ level [16]. Before the origin of this discrepancy is found it will be hard to make further progress in pinning down theoretical uncertainties.

- In this context iso-spin breaking effects in the relationship between the $\tau$ - and the $e^{+} e^{-}$-data have been extensively investigated in [1718]. The question remains whether all possible iso-spin violating effects have been taken into account in which case the discrepancy would have to be attributed to experimental problems.

- A new bound $\delta a_{\mu}(0.6-2.0 \mathrm{GeV})<0.7 \times$ $10^{-10} 19$ for the contributions of $\pi \pi \gamma, \pi \eta \gamma$ which include decay products from $\pi^{0} \gamma, \sigma \gamma, f \gamma, a_{1} \gamma$ yields a severe constraint on possible missing contributions reported elsewhere 2021].

\section{Ongoing activities:}

1) $R$ from radiative return at meson factories:

- New results for hadronic $e^{+} e^{-}$cross-sections are expected soon from KLOE, BABAR and BELLE. These experiments, running at fixed energies, are able to perform measurements via the radiative return method 222324252627 . Preliminary results presented recently by KLOE agree very well with the final CMD-2 $e^{+} e^{-}$ data 25. KLOE is close to finalizing results for the $\pi \pi$ channel. BABAR has presented interesting preliminary results, in particular on the $4 \pi$ channels 26. The feasibility of such crosssection measurements has also been studied at BELLE [27].

\section{2) $R$ from energy scans:}

- $R$ measurements at CLEO [28] will resolve the Mark I vs. CB "discrepancy". Measurements at the energies 7.0, 7.4, 8.4, 9.410 .0 and $10.3 \mathrm{GeV}$ will be performed.

Future plans:

- An upgrade of BEPCI/BESII is in progress: 
BEPCII/BESIII will run at energies 2.2, 2.6 and $3.0 \mathrm{GeV}$ and measure $R$ at precisions 5.5, 3.4 and $3.4 \%$ ([7.6, 7.0 and 5.6] \% now), respectively [8].

- The upgrade of VEPP-2M to VEPP-2000 is in progress and will allow to measure $R$ in the energy range $0.4-2.0 \mathrm{GeV}$ in the years $2005-2010$. The luminosity will increase by a factor of 10 , the detectors CMD-2 and SND should gain a factor of 2 in precision [29]. This will provide important improvements for the presently most problemetic energy range 1.4 - $2 \mathrm{GeV}$.

- At DA $\Phi$ NE an upgrade to $2 \mathrm{GeV}$ for a $R$-scan is under discussion 30.

\section{Theory issues:}

- Last but not least an important change in the hadronic contribution to $a_{\mu}$ was the change in sign of the leading hadronic light-by-light contribution $\left(\pi^{0}\right.$ exchange) 31323334 .

- Progress was made also in calculating the radiative corrections to $\pi^{+} \pi^{-}$production for energy scans, for inclusive measurements in radiative return [3536] and in photon tagging [22 23 37] relevant at the meson $(\Phi, B)$ factories.

Some of these results have substantially influenced the precision of the evaluations of the hadronic VP contribution to $\alpha_{\mathrm{QED}}\left(M_{Z}\right)$ and $(g-$ $2)_{\mu}$ since 1995 38. The present status is reviewed in the following. All of the forthcoming measurements are able to substantially improve future evaluations.

Note concerning future developments: In order to get $\sigma_{\text {had }}$ at a precision better than $1 \%$ in regions where data must be used in the dispersion integrals, the following aspects should be keep in mind. Also in future two different methods for measuring low energy hadronic cross sections will be applied: the enegy scan and the radiative return or photon tagging method. Both methods have their own advadtages and problems and require appropriate efforts on the theory side. The photon tagging method is one power in $\alpha$ higher order and requires a correspondingly higher effort on the theory side. The lower cross section is compensated for by the dramatically enhanced cross section at the resonsnce at which the maschine is running. Cross checks of results from both methods are important. Specific problems are encountered at very low energies, where the total cross section only can be obtained by adding up exclusive channel measurements (particle identification etc.). In a transition region at medium energies one urgently requires cross checking of exclusive vs. inclusive measurements. At higher energy finally inclusive measurements are done and simplify live considerably. Theory has to improve on radiative corrections for Bhabha scattering (small/wide angle) as a reference process for $\mu^{+} \mu^{-}$for normalization/cross check/event separation and finally the hadronproduction $\pi^{+} \pi^{-}, \ldots$ itself. Important questions concern: How to estimate the error from missing higher order? How to estimate model dependence of final state radiation (FSR) from hadrons? Do we have background under control? e.g.: $e^{+} e^{-} \rightarrow e^{+} e^{-} \pi^{+} \pi^{-}$in inclusive measurement of $\pi^{+} \pi^{-}$is at $1-2 \%$ level $^{4}$ at low invariant $M_{\pi \pi}^{2}$. Let me point out that the presently aimed precision requires to check the proper treatment of VP subtraction: we now need to subtract the true time-like $\alpha(s)$ in $\mathrm{s}$-channels. In Bhabha scattering programs one has to carefully distinguish t-channel $(\alpha(t))$ and s-channel $(\alpha(s))$ subtractions. One lesson we have learned in particular from precision physics at LEP/SLC: we in any case need dedicated comparisons of different calculations/generators, in order to make sure that things are under control. See also the contributions 374041. A serious problem remains the radiation of photons by the final state hadrons, which we cannot calculate reliably. Models like scalar QED for the photon radiation by pions decsribe well soft real and virtual photons but fail at some point to model hard real and virtual photons. The problem is particularly serious for charged current processes like $\tau$-decay (see below) where large UV logs come into play. For steps towards a systematic treatment within the effective Lagrangian approach see 171842 .

More details on theoretical aspects of radiative return measurements of $R$ are discussed in 43]. For more information on the status and prospects of $R$ measurements see 44 .

\footnotetext{
${ }^{4}$ In Ref. 35 the high energy approximation has been considered which overestimates the effect, as has been pointed out in 39, recently.
} 


\section{EVALUATION OF $\alpha\left(M_{Z}\right)$}

The non-perturbative hadronic contribution $\Delta \alpha_{\text {had }}^{(5)}(s)$ to the photon vacuum polarization $\Delta \alpha=\Pi_{\gamma}^{\prime}(0)-\Pi_{\gamma}^{\prime}(s)$ can be evaluated in terms of $\sigma\left(e^{+} e^{-} \rightarrow\right.$ hadrons $)$ data via a dispersion relation

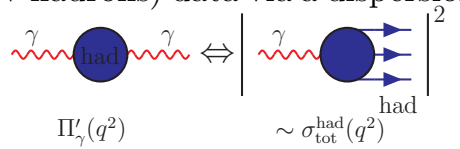

which may be written as

$$
\begin{aligned}
\Delta \alpha_{\text {had }}^{(5)}(s)=- & \frac{\alpha s}{3 \pi}\left(\oint_{4 m_{\pi}^{2}}^{E_{\text {cut }}^{2}} d s^{\prime} \frac{R_{\gamma}^{\mathrm{data}}\left(s^{\prime}\right)}{s^{\prime}\left(s^{\prime}-s\right)}\right. \\
& \left.+\oint_{E_{\mathrm{cut}}^{2}}^{\infty} d s^{\prime} \frac{R_{\gamma}^{\mathrm{pQCD}}\left(s^{\prime}\right)}{s^{\prime}\left(s^{\prime}-s\right)}\right)
\end{aligned}
$$

with $R_{\gamma}(s) \equiv \sigma^{(0)}\left(e^{+} e^{-} \rightarrow \gamma^{*} \rightarrow\right.$ hadrons $) / \frac{4 \pi \alpha^{2}}{3 s}$. My update for $M_{Z}=91.19 \mathrm{GeV}$ utilizing $R(s)$ data up to $\sqrt{s}=E_{\text {cut }}=5 \mathrm{GeV}$ and for the $\Upsilon$ resonance-region between 9.6 and $13 \mathrm{GeV}$ and perturbative QCD from 5.0 to $9.6 \mathrm{GeV}$ and for the high energy tail above $13 \mathrm{GeV}$ yields

$$
\begin{aligned}
\Delta \alpha_{\text {hadrons }}^{(5)}\left(M_{Z}^{2}\right) & =0.027690 \pm 0.000353 \\
\alpha^{-1}\left(M_{Z}^{2}\right) & =128.936 \pm 0.048 .
\end{aligned}
$$

With more theory input, based on the Adler function method (see below), we obtain (see Fig. 11)

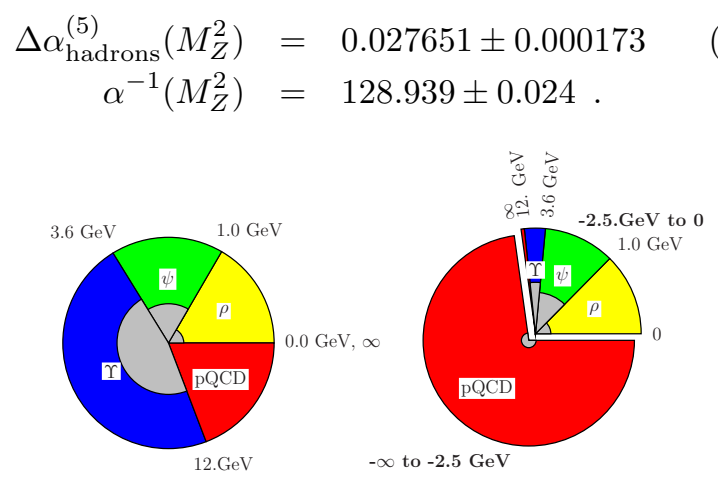

Figure 1. Comparison of the distribution of contributions and errors (shaded areas scaled up by 10) in the standard (left) and the Adler function based approach (right), respectively.
Other recent evaluations agree well with these results, although the methods are quite different in spirit:

$$
\begin{aligned}
\Delta \alpha_{\text {hadrons }}^{(5)}\left(M_{Z}^{2}\right)= & 0.02768 \pm 0.00036[45] \\
& 0.02769 \pm 0.00018[\underline{46}
\end{aligned}
$$

(see also 346]). Other aspects concerning $\alpha\left(M_{Z}\right)$ were discussed in [4748] (see also [49]).

\section{EVALUATION OF $a_{\mu} \equiv(g-2)_{\mu} / 2$}

The leading non-perturbative hadronic contribution to $a_{\mu}^{\text {had }}$, given by the diagram

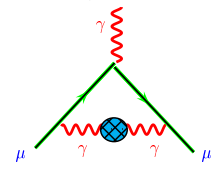

can be obtained again in terms of $R_{\gamma}(s)$ via the dispersion integral

$$
\begin{aligned}
a_{\mu}^{\mathrm{had}}=\left(\frac{\alpha m_{\mu}}{3 \pi}\right)^{2}\left(\int_{4 m_{\pi}^{2}}^{E_{\text {cut }}^{2}} d s \frac{R_{\gamma}^{\mathrm{data}}(s) \hat{K}(s)}{s^{2}}\right. \\
\left.+\int_{E_{\mathrm{cut}}^{2}}^{\infty} d s \frac{R_{\gamma}^{\mathrm{pQCD}}(s) \hat{K}(s)}{s^{2}}\right)
\end{aligned}
$$

where $\hat{K}(s) \in[0.63 \cdots, 1.0]$ is a bounded monotonically increasing function.

Again the experimental error of $R$ implies the main theoretical uncertainty in the prediction of $a_{\mu}$. Since the low energy contribution is enhanced by $1 / s^{2}$, about $\sim 67 \%$ of the error on $a_{\mu}^{\text {had }}$ comes from the low energy region $4 m_{\pi}^{2}<m_{\pi \pi}^{2}<M_{\Phi}^{2}$. This is why the $\rho$ resonance region dominated by the $e^{+} e^{-} \rightarrow \pi^{+} \pi^{-}$channel plays a prominent role in a precise prediction of $a_{\mu}^{\text {had }}$. My $e^{+} e^{--}$ data based update yields (see Fig. 22)

$a_{\mu}^{\text {had(1) }}=(694.8 \pm 8.6) \times 10^{-10}$.

With this estimate I get

$$
\begin{aligned}
a_{\mu}^{\text {the }}= & \left(11659179.4 \pm 8.6_{\mathrm{had}} \pm 3.5_{\mathrm{LBL}}\right. \\
& \left. \pm 0.4_{\mathrm{QED}+\mathrm{EW}}\right) \times 10^{-10}
\end{aligned}
$$

${ }^{5}$ Other recent $e^{+} e^{-}$-data based results in units of $10^{-10}$ are $684.7 \pm 7.0$ [16] and $683.1 \pm 6.2$ [46]. The $\tau$-data based result is [16] $a_{\mu}^{\text {had(1) }}=(701.9 \pm 6.1) \times 10^{-10}$. 
which compares to the most recent experimental result 5051

$\left.a_{\mu}^{\exp }=11659203 \pm 8\right) \times 10^{-10}$.

The "discrepancy" $\left|a_{\mu}^{\text {the }}-a_{\mu}^{\exp }\right|=(23.6 \pm 12.3) \times$ $10^{-10}$ corresponds to a deviation of about $1.9 \sigma$. For other recent estimates see 16524653 . The status of the theory has been reviewed in 31. For a review of the history of $a_{\mu}$ experiments see [54].

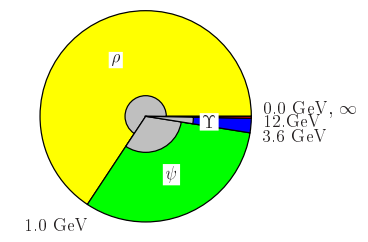

Figure 2. The distribution of contributions and errors (shaded areas scaled up by 10) for $a_{\mu}^{\text {had }}$.

Some comments: What we need for inserting into the dispersion integrals is on the one hand the one particle irreducible (1pi) "blob", which corresponds to the VP undressed cross-section (pion form factor)

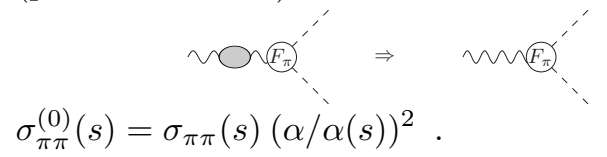

On the other hand, the hadronic 1pi "blob" should include photonic corrections:

$$
\text { had } \sim+n \sum_{\text {had }}^{n^{\gamma}} \sim+\cdots
$$

Thus one has to add the theoretical prediction for FS radiation (including full photon phase space):

$\sigma_{\pi \pi}^{(\gamma)}(s)=\sigma_{\pi \pi}^{(0)}(s)\left(1+\eta(s) \frac{\alpha}{\pi}\right)$

to order $O(\alpha)$, where $\eta(s)$, to the extent that scalar QED (sQED) for the pion photon interactions is assumed as a model, is a known correction factor (Schwinger 1989). The corresponding $O(\alpha)$ contribution to the anomalous magnetic moment of the muon is

$\delta^{\gamma} a_{\mu}^{\text {had }}=(38.6 \pm 1.0) \times 10^{-11}$.

In order to get a model independent result one has to measure FSR [55, preferably in an energy scan experiment.
When including or excluding (note that only more or less hard real photons can be cut away) final state photons (FSR) one has to be aware of the Kinoshita, Lee, Nauenberg (KLN) theorem at work. The fully inclusive cross-section, including virtual $(V)$, soft $(S)$ and all hard $(H)$ photons, is of the form $\sigma_{0}\left(1+\frac{\alpha}{\pi} C\right) \quad C$ a constant of $O(1)$ like $3 / 4$ for $\mu$-pair, 3 (in sQED) for $\pi$-pair production, i.e. typically this correction is small (and positive) as a matter of cancellations between $V+S$, on the one hand, and $H$, on the other hand. A typical size of the FSR contribution is $\sim .2 \%$. Exclusive quantities, in contrast, involve large logs on the photon cut energy as well as collinear logs. According to Bloch and Nordsieck, $V+S$ cannot be separated (separately IR divergent) and there are potentially large logs proportional to $\ln \left(\sqrt{s} / E_{\gamma \text { cut }}\right)$ and $\ln \left(s / m_{\pi}^{2}\right)$ which multiply $\frac{\alpha}{\pi}$ and the complementary hard photon part (integrated over full phase space) $H$ exhibits the same $\operatorname{logs}$ as $V+S$ but precisely of opposite sign. All logs cancel in the sum (inclusive measurement). Consequences are the following: 1) cutting out all hard photons and subtracting $V+S$ using sQED one expects a $.5 \%$ model ambiguity (sQED vs. fQED) 2) adding the missing $H$ part using the same model (as used for subtracting $V+S$ ) makes the correction small (for any model) and thus also reduces the model ambiguity to about .1\% (sQED vs. fQED) [55]. The true model error of course cannot be obtained this way.

As mentioned before the low energy region plays a particularly important role for the evaluation of $a_{\mu}^{\text {had }}$. The region is dominated by the pion form factor $F_{\pi}(s)$, for which severe theory constraints exist. In fact analyticity, unitarity and the chiral limit relate space-like data, $\pi \pi$-scattering phase shifts and time-like data in a stringent way (Omnès-Muskhelishvili approach). Work in progress will allow us to get a substantially improved low energy contribution [565357]. For earlier attempts in this direction see [5859]. 


\section{5. $e^{+} e^{-}$-CROSS SECTIONS VIA $\tau$-DECAY SPECTRAL FUNCTIONS}

To the extent that the charged vector current is (approximately) conserved (CVC), the iso-vector part of $\sigma\left(e^{+} e^{-} \rightarrow\right.$ hadrons) may be obtained by an iso-spin rotation from $\tau$-decay spectra. The precise relation derives from the diagrams

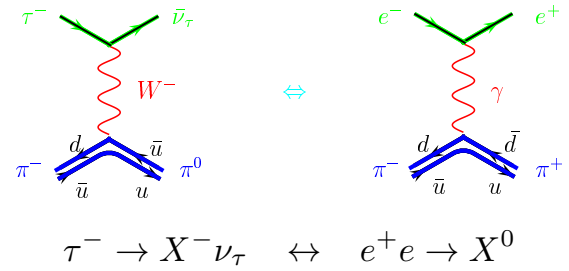

where $X^{-}$and $X^{0}$ are hadronic states related by an iso-spin rotation. The $e^{+} e^{-}$cross-section is then given by

$\sigma_{e^{+} e^{-} \rightarrow X^{0}}^{I=1}=\frac{4 \pi \alpha^{2}}{s} v_{1, X^{-}}, \quad \sqrt{s} \leq M_{\tau}$

in terms of the $\tau$ spectral function $v_{1}$. In principle, the such enhanced " $e^{+} e^{-}$data set" is able to improve substantially the knowledge of the $\pi^{+} \pi^{-}$ channel ( $\rho$-resonance contribution) which is the dominating contribution $(72 \%)$ to $a_{\mu}^{\text {had }}$. Note that the $\tau$-data can replace $e^{+} e^{-}$-data only partially, because the $I=1$ part only accounts for about $75 \%$ of the total, summed over all channels. The inclusion of the $\tau$ data (from ALEPH) has been pioneered in Ref. 14] at a time when errors of the CMD-2 data were a factor of two larger and there was no reason not to combine the $e^{+} e^{-}$ and $\tau$ data. In particular for $a_{\mu}^{\text {had }}$ the reduction of the error was about $30 \%: \delta a_{\mu}: 15.6 \times 10^{-10} \rightarrow$ $10.2 \times 10^{-10}$ while $\delta \Delta \alpha: 0.00067 \rightarrow 0.00065$.

In the meantime, CMD-2 was able to substantially reduce the systematic error in $e^{+} e^{-} \rightarrow$ $\pi^{+} \pi^{-}$and OPAL and CLEO came up with independent measurements of $\tau$ spectral functions. Obviously, before data can be combined all kind of iso-spin breaking effects have to be taken into account. Taking into account the established isospin breaking effects [17] a comparison of the $\tau$ data is shown in Fig. [3 As already mentioned, the reanalysis based on the most recent data revealed a substantial discrepancy between the $e^{+} e^{-}$and the $\tau$ data to an extent which made a combination of the data impossible [16].

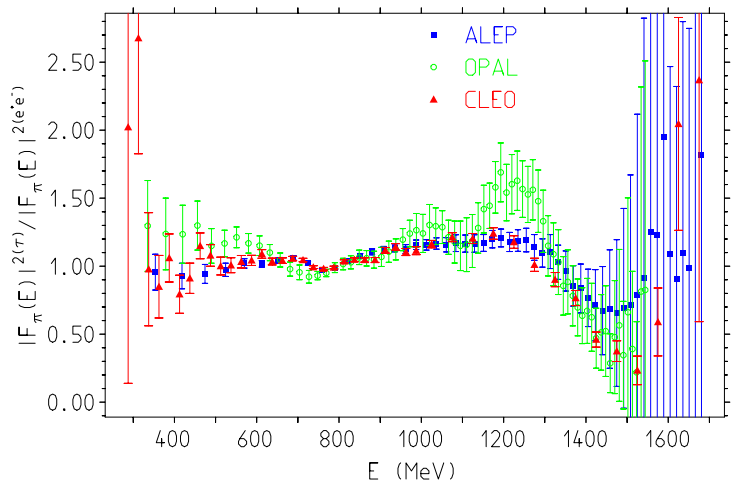

Figure 3. Comparison of the $\tau$-data from ALEPH, CLEO and OPAL, normalized to the CMD-2 fit. Note that the agreement between ALEPH/CLEO and OPAL is not very good.

What may be the origin of the large discrepancy? This question has been analyzed recently in 60. For comparing $e^{+} e^{-}$and $\tau$ data we may use a Gounaris-Sakurai (GS) parametrization of the pion form-factor. It is crucial to compare "commensurate" data:

1) for $e^{+} e^{-}$subtract VP, subtract FSR, undo $\rho-\omega$-mixing and fit for $m_{\rho^{0}}$ and $\Gamma_{\rho^{0}}$ at fixed background (i.e. all other parameters of the GS parametrization held fixed) which yields 60.

$$
m_{\rho^{0}}=773.1 \pm 0.6 \quad, \quad \Gamma_{\rho^{0}}=146.4 \pm 1.0
$$

2) apply the iso-spin corrections 1761

$$
r_{\mathrm{IB}}(s)=\frac{1}{G_{\mathrm{EM}}(s)} \frac{\beta_{\pi^{-} \pi^{+}}^{3}}{\beta_{\pi^{-} \pi^{0}}^{3}} \frac{S_{\mathrm{EW}(\text { old })}}{S_{\mathrm{EW}(\text { new })}}
$$

to the $\tau$ data and fit the corrected $\tau$ data for $m_{\rho^{-}}$ and $\Gamma_{\rho^{-}}$at fixed background, which yields

$$
m_{\rho^{-}}=775.8 \pm 0.7 \quad, \quad \Gamma_{\rho^{-}}=147.8 \pm 1.0 .
$$

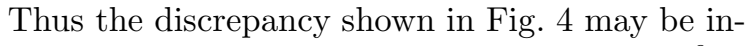
terpreted as a shift in the mass and the width ${ }^{6}$ of the $\rho$ as the leading effect [60] (see also [12]).

Since both data set may be fitted rather well by a GS-formula, it is not very surprising that one can easily fit the ratio to unity within errors by letting $m_{\rho^{-}}$and $\Gamma_{\rho^{-}}$float in the numerator. The

${ }^{6}$ We have $\Delta m_{\rho}=2.7 \pm 0.9[3.1 \pm 0.9] \mathrm{MeV} ; \Delta \Gamma_{\rho}=1.4 \pm$ $1.4[1.8 \pm 1.6] \mathrm{MeV}$, in brackets the values from [12]. 


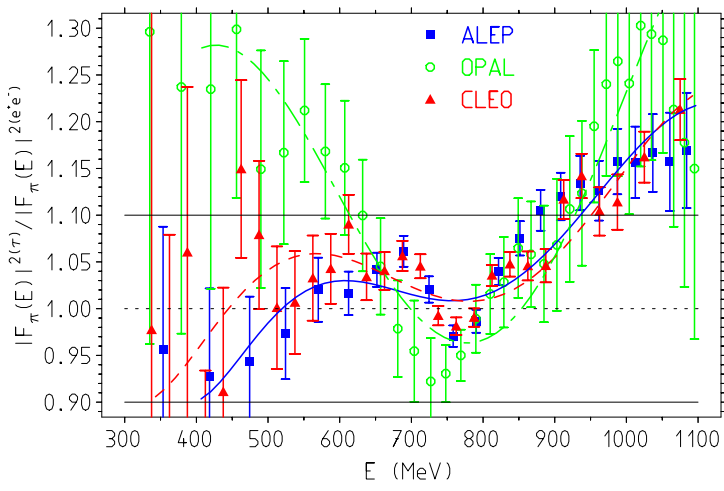

Figure 4. The ratio between $\tau$-data sets from ALEPH, OPAL and CLEO and the $I=1$ part of the CMD-2 fit of the $e^{+} e^{-}$-data. The curves which should guide the eye are fits of the ratios using 8th order Tschebycheff polynomials.

fit yields back $m_{\rho^{0}}$ and $\Gamma_{\rho^{0}}$; this is not completely trivial as it works over the whole range shown. Appraisal of the result:

- We now have two sets of reasonably consistent data: ALEPH and CLEO $\tau$-data vs. CMD-2 and $\operatorname{KLOE} e^{+} e^{-}$-data.

- What effect is able to to give $10 \%$ in a resonance tail? Answer: a shift of the energy by about $1 \%$. This could be a problem of energy calibration, but this is very unlikely. Rather the physical resonance parameters have no reason to be identical!

- In spite of possible experimental problems, there is no reason to expect the neutral channel parameters to be the same as the charged current ones. Example, pions: $m_{\pi^{ \pm}}-m_{\pi^{0}}=$ $4.5935 \pm 0.0005 \mathrm{MeV}$. Why should this be very different for the $\rho$ a similar (same quark content) bound state? I do not think that the Goldstoneboson nature of the pions necessarily makes such an iso-spin breaking very different.

Our conclusion:

- the observed discrepancy is a so far unaccounted iso-spin breaking effect, which the $\tau$-data have to be corrected for!

- Relevant for calculating $a_{\mu}^{\text {had }}$ are the $e^{+} e^{-}$-data in first place.

- How to include the $\tau$-data then? One should know $\Delta m_{\rho}$ and $\Delta \Gamma_{\rho}$ from elsewhere. Other parameters like $m_{\rho^{\prime}}$ and $\Gamma_{\rho^{\prime}}$ are affected as well, but his is a higher order effect.

- In spite of the fact that the peak has to be shifted downwards, $a_{\mu}^{\text {had }}$ does not increase as naively expected, in view of the $s^{-2}$ weight in the integral. As it should be, it rather decreases, because the width also substantially goes down and over-compensates the effect of the mass shift.

- If you don't like the idea: notice that the $\frac{\partial a_{\mu}^{\text {had }}}{\partial m_{\rho}\left[\Gamma_{\rho}\right]}$ is very large so that this quantity is very sensitive to the parameters. If it is not a true iso-spin breaking effect, it would have to be an energy calibration problem.

My conclusion: we are back to one prediction for $a_{\mu}$ at $2 \sigma$ from the experimental value!

\section{CONTROLLING PQCD VIA THE ADLER FUNCTION}

In particular for $\Delta \alpha^{\text {had }}$ the standard evaluation based on data is very sensitive to errors of the data up to the $\Upsilon$ energies. For future needs in precision physics, new experiments are needed which measure $\sigma_{\text {had }}$ at the $1 \%$ level up to $10 \mathrm{GeV}$. Is it possible to depend less on inaccurate data and to use perturbative QCD instead? The so called "theory driven" approach 626364656646 relies on replacing to a large extent the measured $R(s)$ by its perturbatively calculated version (see [67]). The quality of such a procedure is hard to control beyond some perturbative windows, because of non-perturbative effects present in $R(s)$ (resonances, thresholds). An alternative approach, which allows us to control the applicability of pQCD in a much better way was proposed long time ago by Adler: the Euclidean (space-like) method via the Adler function

$$
\begin{aligned}
D(-s) & \doteq \frac{3 \pi}{\alpha} s \frac{d}{d s} \Delta \alpha_{\mathrm{had}}(s)=-\left(12 \pi^{2}\right) s \frac{d \Pi_{\gamma}^{\prime}(s)}{d s} \\
D\left(Q^{2}\right) & =Q^{2} \int_{4 m_{\pi}^{2}}^{\infty} d s \frac{R(s)}{\left(s+Q^{2}\right)^{2}} .
\end{aligned}
$$

It is an absolutely smooth function which can be easily calculated from the experimental data on the one hand and by pQCD on the other hand. In Fig. [5 the present status is shown.

We see that in the space-like approach pQCD works well for $Q^{2}=-q^{2}>2.5 \mathrm{GeV}$. There we may replace the experimental Adler function by 


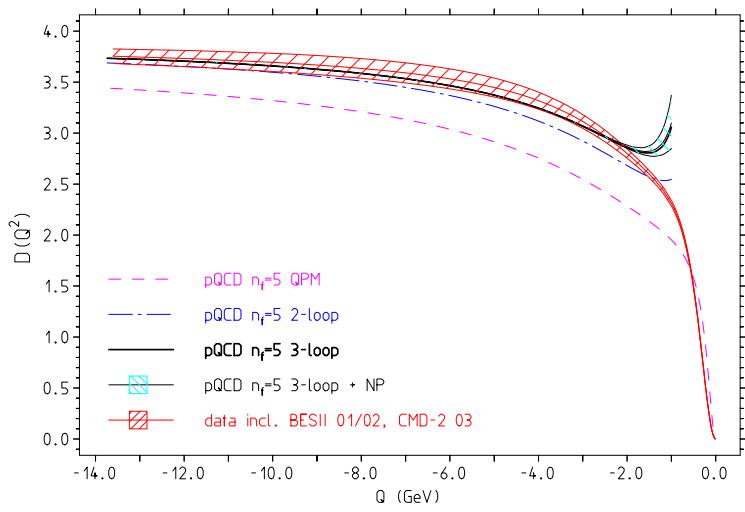

Figure 5. "Experimental" Adler-function versus theory (pQCD + NP).

the pQCD one ${ }^{7}$.

In this approach we thus may calculate

$$
\begin{array}{r}
\Delta \alpha_{\text {had }}\left(-Q^{2}\right) \sim \frac{\alpha}{3 \pi} \int d Q^{\prime 2} \frac{D\left(Q^{\prime 2}\right)}{Q^{\prime 2}} \\
\begin{aligned}
\Delta \alpha_{\text {had }}^{(5)}\left(-M_{Z}^{2}\right)= & {\left[\Delta \alpha_{\text {had }}^{(5)}\left(-M_{Z}^{2}\right)-\Delta \alpha_{\text {had }}^{(5)}\left(-s_{0}\right)\right]^{\mathrm{pQCD}} } \\
& +\Delta \alpha_{\text {had }}^{(5)}\left(-s_{0}\right)^{\text {data }}
\end{aligned}
\end{array}
$$

and obtain, for $s_{0}=(2.5 \mathrm{GeV})^{2}$ :

$\Delta \alpha_{\text {had }}^{(5)}\left(-s_{0}\right)^{\text {data }}=0.007417 \pm 0.000086$

$\Delta \alpha_{\text {had }}^{(5)}\left(-M_{Z}^{2}\right)=0.027613 \pm 0.000086 \pm 0.000149$.

The second error comes from the variation of the pQCD parameters. The largest uncertainty is due to the poor knowledge of the charm mass. I have taken errors to be $100 \%$ correlated here.

Link between space-like and time-like region is the difference

$$
\Delta \alpha_{\text {had }}^{(5)}\left(M_{Z}^{2}\right)-\Delta \alpha_{\text {had }}^{(5)}\left(-M_{Z}^{2}\right)=0.000038 \pm 0.000005
$$

which can be calculated in pQCD. Altogether we obtain the value given earlier in (5). For more details I refer to 6869.

The result clearly demonstrates how important the precise knowledge of the QCD parameters

\footnotetext{
${ }^{7}$ Note that in the time-like approach pQCD works well only in the "perturbative windows" $3.00-3.73 \mathrm{GeV}, 5.00$ $-10.52 \mathrm{GeV}$ and $11.50-\infty 67$.
}

is. In the Adler function approach the error is dominated by the parameter uncertainties in the pQCD part (see Fig.11). Recent progress achieved by $\mathrm{pQCD} / \mathrm{SR}$ (sum rules, moment method) and lattice QCD (LQCD) is summarized in the following Table 67 70 71]: In LQCD in not too far future full QCD results [unquenched] extrapolated to the continuum limit will be available.

\section{STATUS AND OUTLOOK}

Conclusions: in addition to planned upgrades which have been reported, a $\tau$-charm factory would be needed which should be able to perform an energy scan between 2 and $3.6 \mathrm{GeV}$ with $1 \%$ accuracy. This would help to satisfy requirements of future precision experiments g-2, GigaZ, etc.

Last but not least: we need further progress in theory

- more careful study of iso-spin breaking in $\tau$ data vs. $e^{+} e^{-}$-data

- constraints to $F_{\pi}(s)$ from $\chi \mathrm{PT}$, unitarity and analyticity below the about $2 M_{K}$.

- still a theoretical challenge: the hadronic lightby-light scattering contribution!

- Concerning the $\tau$-data vs. $e^{+} e^{-}$-data discrepancy: we need more careful check of radiative corrections (FSR, in particular) which have been applied!

- Further progress in radiative corrections calculations is needed for the processes involved in $R$-measurements

- Also further progress in determination of the QCD parameters is indispensable.

The big experimental challenge: one has to attempt cross-section measurements at the $1 \%$ level up to $J / \psi$ [or even up to $\Upsilon$ ].

\section{Acknowledgments}

I thank the organizers of the workshop SIGHAD03 for the kind invitation and hospitality at Pisa. Thanks also to Mikhail Kalmykov for helpful discussions and for reading the manuscript.

\section{REFERENCES}

1. J. Alcaraz et al. [LEP Collaborations], LEPEWWG/2003-01; D. Abbaneo et al. 
Table 1

\begin{tabular}{c|ccccc}
\hline \hline Ref & $\alpha_{s}\left(M_{Z}\right)$ & $\Lambda_{\frac{N_{f}}{\mathrm{MS}}=0}[\mathrm{MeV}]$ & $m_{s}\left(m_{s}\right)[\mathrm{MeV}]$ & $m_{c}\left(m_{c}\right)[\mathrm{GeV}]$ & $m_{b}\left(m_{b}\right)[\mathrm{GeV}]$ \\
\hline PDG & $0.118(3)$ & - & $80-155$ & $1.0-1.4$ & $4.0-4.5$ \\
67] & $0.124_{-0.014}^{+0.011}$ & - & - & $1.304(27)$ & $4.191(51)$ \\
70] & - & - & - & $1.3(1)$ & $4.2(1)$ \\
71] & - & $238(19)[\mathrm{Q}]$ & $97(4)[\mathrm{Q}]$ & $1.301(34)[\mathrm{Q}]$ & $4.12(7)(4)[\mathrm{Q}]$ \\
\hline
\end{tabular}

New results for QCD parameters. [Q] quenched results from LQCD

[LEP Collaborations], LEPEWWG/2002-02, hep-ex/0212036

2. Proc. of the Mini-Workshop on "Electroweak Precision Data And The Higgs Mass", Zeuthen 2003, Eds. S. Dittmaier and K. Mönig, K. Mönig, hep-ph/0308133 and references therein.

3. B. Pietrzyk, "Fit of S.M., results on $\alpha_{\mathrm{em}}\left(M_{Z}\right)$ and influence on the Higgs mass", these proceedings.

4. R. R. Akhmetshin et al. [CMD-2 Collaboration], hep-ex/0308008

5. G. Fedotovitch, "Precise measurements of hadronic cross section at VEPP-2M", these proceedings.

6. R. R. Akhmetshin et al. [CMD-2 Collaboration], Phys. Lett. B 527 (2002) 161.

7. J. Z. Bai et al. [BES Collaboration], Phys. Rev. Lett. 84 (2000) 594; Phys. Rev. Lett. 88 (2002) 101802.

8. H. Hu, "Status and future plans of $R$ measurement at BES", these proceedings.

9. R. Barate et al. [ALEPH Collaboration], Z. Phys. C 76 (1997) 15; Eur. Phys. J. C 4 (1998) 409.

10. K. Ackerstaff et al. [OPAL Collaboration], Eur. Phys. J. C 7 (1999) 571.

11. S. Anderson et al. [CLEO Collaboration], Phys. Rev. D 61 (2000) 112002.

12. M. Davier, " $\tau$ spectral functions and QCD", these proceedings.

13. Y. S. Tsai, Phys. Rev. D 4 (1971) 2821 [Erratum-ibid. D 13 (1976) 771].

14. R. Alemany, M. Davier and A. Höcker, Eur. Phys. J. C 2 (1998) 123.

15. H. Hayashii, " $\tau \rightarrow 2 \pi$ decay mode from Belle", these proceedings.

16. M. Davier, S. Eidelman, A. Höcker and
Z. Zhang, Eur. Phys. J. C 27 (2003) 497; hep-ph/0308213

17. V. Cirigliano, G. Ecker and H. Neufeld, Phys. Lett. B 513 (2001) 361; JHEP 0208 (2002) 002.

18. G. Ecker, "Isospin violating and radiative corrections to $\tau \rightarrow 2 \pi$ decay", these proceedings.

19. S. Eidelman, private communication.

20. S. Narison, Phys. Lett. B 568 (2003) 231.

21. S. Dubnicka, these proceedings.

22. S. Binner, J. H. Kühn and K. Melnikov, Phys. Lett. B 459 (1999) 279; H. Czyż and J. H. Kühn, Eur. Phys. J. C18 (2001) 497; G. Rodrigo, H. Czyż, J. H. Kühn and M. Szopa, Eur. Phys. J. C 24 (2002) 71; J. H. Kühn and G. Rodrigo, Eur. Phys. J. C 25 (2002) 215; H. Czyż, A. Grzelinska, J. H. Kühn and G. Rodrigo, Eur. Phys. J. C 27 (2003) 563; hep-ph/0308312

23. V. A. Khoze et al., Eur. Phys. J. C 25 (2002) 199.

24. A. Aloisio et al. [KLOE Collaboration], hep-ex/0307051; A. Denig [KLOE Collaboration], hep-ex/0311012.

25. B. Valeriani, "Results on $R$ from KLOE", these proceedings.

26. M. Davier, "Results on $R$ from BABAR", these proceedings.

27. S. Eidelman, "First results on $R$ from BELLE", these proceedings.

28. S. Dytman, "Results and plans on $R$ from CLEO", these proceedings.

29. S. Eidelman, "Plans on $R$ with VEPP-2000", these proceedings.

30. A. Denig, "Future plans for Dafne", these proceedings.

31. A. Nyffeler, hep-ph/0305135 "Theory of ( $g-$ 2) $\mu$ ", these proceedings. 
32. M. Knecht and A. Nyffeler, Phys. Rev. D 65 (2002) 073034; M. Knecht, A. Nyffeler, M. Perrottet and E. De Rafael, Phys. Rev. Lett. 88 (2002) 071802.

33. J. Bijnens, E. Pallante and J. Prades, Nucl. Phys. B 474 (1996) 379; Nucl. Phys. B 626 (2002) 410.

34. M. Hayakawa and T. Kinoshita, Phys. Rev. D 57 (1998) 465 [Erratum-ibid. D 66 (2002) 019902].

35. A. Hoefer, J. Gluza and F. Jegerlehner, Eur. Phys. J. C 24 (2002) 51.

36. A. B. Arbuzov et al., hep-ph/0308292

37. G. Rodrigo, "Radiative return at meson factories", these proceedings.

38. S. Eidelman and F. Jegerlehner, Z. Phys. C 67 (1995) 585; see also: F. Jegerlehner, Z. Phys. C 32 (1986) 195; Nucl. Phys. Proc. Suppl. 51C (1996) 131; J. Phys. G 29 (2003) 101.

39. H. Czyż and E. Nowak, hep-ph/0310335

40. A. Sibidanov, "Radiative corrections at CMD-2", these proceedings.

41. C.M. Carloni Calame, "The BABAYAGA event generator", these proceedings.

42. I. Scimemi, "E.M. in hadronic processes", these proceedings.

43. J. Kühn, "Status and prospects of MC tools for ISR", these proceedings.

44. Z. Zhao, "Status and prospects of $R$ measurements", these proceedings.

45. H. Burkhardt and B. Pietrzyk, Phys. Lett. B 513 (2001) 46.

46. K. Hagiwara et al., Phys. Lett. B 557 (2003) 69; T. Teubner, "Hadronic contributions to $a_{\mu} "$, these proceedings.

47. L. Trentadue, "The running of $\alpha_{\mathrm{QED}}$ in small angle Bhabha scattering", these proceedings.

48. G. Degrassi and A. Vicini, hep-ph/0307122 A. Vicini, "Two-loop renormalization of the electric charge in the Standard Model", these proceedings.

49. F. Jegerlehner and J. Fleischer, Phys. Lett. B 151 (1985) 65; Acta Phys. Polon. B 17 (1986) 709; F. Jegerlehner, hep-ph/0105283

50. G. W. Bennett et al. [Muon g-2 Collaboration], Phys. Rev. Lett. 89 (2002) 101804 [Erratum-ibid. 89 (2002) 129903].
51. B. Lee Roberts, "Results and prospects on the measurement of $(g-2)_{\mu}$ ", these proceedings.

52. M. Davier, "Hadronic contributions to $a_{\mu}$ ", these proceedings.

53. G. Colangelo, "Hadronic contributions to $a_{\mu} "$, these proceedings.

54. F. Farley, "46 years of $g-2$ ", these proceedings.

55. J. Gluza et al., Eur. Phys. J. C 28 (2003) 261.

56. H. Leutwyler, hep-ph/0212324

57. J. Portoles, "The hadronic cross-section in the resonance energy region", these proceedings.

58. J. A. Casas, C. Lopez and F. J. Yndurain, Phys. Rev. D 32 (1985) 736; J. F. De Troconiz and F. J. Yndurain, Phys. Rev. D 65 (2002) 093001.

59. B. V. Geshkenbein, Phys. Rev. D 61 (2000) 033009.

60. S. Ghozzi and F. Jegerlehner, hep-ph/0310181

61. J. Erler, hep-ph/0211345

62. A. D. Martin and D. Zeppenfeld, Phys. Lett. B 345 (1995) 558.

63. M. Davier, A. Höcker, Phys. Lett. B 419, 419 (1998); ibid. Phys. Lett. B 435, 427 (1998).

64. J.H. Kühn, M. Steinhauser, Phys. Lett. B 437, 425 (1998)

65. S. Groote, J.G. Körner, N.F. Nasrallah, K. Schilcher, Phys. Lett. B 440, 375 (1998)

66. J. Erler, hep-ph/9803453

67. J. H. Kühn and M. Steinhauser, Nucl. Phys. B 619 (2001) 588 [Erratum-ibid. B 640 (2002) 415]; M. Steinhauser, "Impact of $\sigma\left(e^{+} e^{-} \rightarrow\right.$ hadrons) measurements on the parameters of the S. M.", these proceedings.

68. S. Eidelman, F. Jegerlehner, A. L. Kataev and O. Veretin, Phys. Lett. B 454 (1999) 369; F. Jegerlehner, hep-ph/9901386

69. F. Jegerlehner, hep-ph/0308117.

70. A. Hoang, "Status of quark masses", these proceedings.

71. M. Della Morte et al. [ALPHA collaboration], hep-lat/0209023

J. Rolf and S. Sint [ALPHA Collaboration], JHEP 0212 (2002) 007; J. Rolf, "Strong coupling and quark masses from lattice QCD", these proceedings. 